\title{
Circ_0045714/miR-331-3p interaction affects IL-1 $\beta$-evoked human articular chondrocyte injury through regulating PIK3R3 in a ceRNA regulatory cascade
}

\author{
Ran Ding ${ }^{1 \dagger}$, Jinsong Zhou ${ }^{2 \dagger}$, Jianguo $\mathrm{Xu}^{2}$, Huajie $\mathrm{Lu}^{2}$, Tingting Zhang ${ }^{2}$, Xiong Xiang ${ }^{2}$ and Zhen $\mathrm{Shi}^{\mathrm{F}^{*}}$
}

\begin{abstract}
Background: Osteoarthritis (OA) is characterized by joint pain and joint function limitation. Hsa_circ_0045714 (circ_0045714) is a novel OA-related circular RNA. However, its repertoire remains to be further clarified in joint chondrocytes.
\end{abstract}

Methods: RNA and protein expression levels and inflammatory factor levels were detected by real-time quantitative polymerase chain reaction, western blotting and enzyme-linked immunosorbent assay. Cell proliferation and apoptosis were determined by colony formation assay, cell counting kit-8 assay and apoptosis assay. Direct interaction was predicted by bioinformatics method and confirmed by dual-luciferase reporter assay.

Results: Expression of circ_0045714 and phosphoinositide-3-kinase (PI3K) regulatory subunit 3 (PIK3R3) was declined, and microRNA (miR)-331-3p was promoted in knee articular cartilages and cells from OA patients, as well as interleukin (IL)-1 $\beta$-challenged human articular chondrocytes (HAC) cell line. In stimulation of IL-1 $\beta$, HAC cells showed a loss of colony formation ability, cell viability and expression of $\mathrm{BCl}-2$ and Collagen II, allied with an increase in apoptosis rate and levels of IL-6, IL-8 and tumor necrosis factor-a, Bcl-2-associated X protein, cleaved caspase-3, and ADAM with thrombospondin motif-5. Noticeably, overexpressing circ_0045714 and inhibiting miR-331-3p could suppress IL-1 $\beta$-evoked these effects, and both were through up-regulating PIK3R3, a key gene in PI3K/AKT signaling pathway. Mechanically, circ_0045714 functioned as competing endogenous RNA (ceRNA) for miR-331-3p and further regulated expression of the downstream target gene PIK3R3.

Conclusion: There was a novel circ_0045714/miR-331-3p/PIK3R3 ceRNA axis in HAC, and its inhibition might be one mechanism of HAC injury in OA.

Keywords: Circ_0045714, miR-331-3p, PIK3R3, IL-13, Human articular chondrocytes, Osteoarthritis

*Correspondence: Dingran417c@163.com

${ }^{\dagger}$ Ran Ding and Jinsong Zhou have contributed equally to this work. 2 Department of Pain1, Hubei Provincial Hospital of Traditional Chinese Medicine, 856 Luoyu Road, Hongshan District, Wuhan City 430070, Hubei Province, China

Full list of author information is available at the end of the article

\section{Introduction}

Osteoarthritis (OA) is a complex multifactorial disease, and its pathology has been advanced in genetics, genomics and epigenetics [1]. Joint pain is highly prevalent in OA patients, and severe pain is the major cause for medical attention and joint replacement [2, 3]. Inflammation has been shown to be associated with the complex etiology of joint pain in OA [4]. Besides, original author(s) and the source, provide a link to the Creative Commons licence, and indicate if changes were made. The images or other third party material in this article are included in the article's Creative Commons licence, unless indicated otherwise in a credit line to the material. If material is not included in the article's Creative Commons licence and your intended use is not permitted by statutory regulation or exceeds the permitted use, you will need to obtain permission directly from the copyright holder. To view a copy of this licence, visit http://creativecommons.org/licenses/by/4.0/. The Creative Commons Public Domain Dedication waiver (http://creativeco mmons.org/publicdomain/zero/1.0/) applies to the data made available in this article, unless otherwise stated in a credit line to the data. 
inflammation-related genes, tumor necrosis factor (TNF)- $\alpha$ and interleukin (IL)- $1 \beta$ are independent predictors for postoperative pain development in $\mathrm{OA}$ patients [5].

One of the major endpoints of OA is the loss of articular cartilage, and chondrocyte is the only cell type in the cartilage [6]. Chondrocytes are activated in the early stage of $\mathrm{OA}$, accompanying with the release of pro-inflammatory cytokines and matrix catabolic enzymes, such as IL- $1 \beta$ and a disintegrin-like and metalloproteinase with thrombospondin motif (ADAMTS)-5 $[7,8]$. Moreover, IL- $1 \beta$ plays versatile roles in different cell types involved in OA pathology, and IL-1 $\beta$-insulted chondrocytes are suitable OA cell models [9]. Noncoding RNAs are new regulatory codes in cartilage development and skeletal disorders including OA [10], as well as the apoptosis and autophagy of chondrocytes [11]. Circular RNAs (circRNAs), a novel type of noncoding RNAs, are ubiquitous, structure-stable, tissue-specific and multifunctional. In OA, circRNAs show diagnostic and therapeutic values in the pathophysiology and treatment [12]. Hsa circ_0045714 (circ_0045714) is a novel circRNA that is differently expressed in fracture and OA $[13,14]$, and it might be a therapeutic target for fracture healing and functional recovery of OA-affected chondrocytes [15, $16]$. However, the role and in-depth molecular mechanism of circ_0045714 in IL-1 $\beta$-induced OA model in chondrocytes are undefined yet.

Phosphoinositide 3-kinase (PI3K) regulatory subunit 3 (PIK3R3) is an inhibitor of PI3K [17] in PI3K/AKT signaling pathway which is closely interwoven with the pathogenesis of OA $[18,19]$. RNA interference is a cellular mechanism for post-transcriptional gene regulation mediated by small interfering RNAs (siRNAs) and microRNAs (miRNAs) [20]. SiRNAs, a doublestranded RNA molecule with about 20 nucleotides, participate in musculoskeletal disorders and tender homeostasis and healing [21, 22]. MiRNAs are small noncoding RNAs that are useful for diagnostic or management purposes in both OA and tendon injuries [23, 24]. However, RNA interference-based regulation of PIK3R is seldom clarified yet. MiRNA (miR)-331-3p is one of miRNAs that could inactivate this pathway in carcinogenesis process $[25,26]$. Nevertheless, whether there is an interaction between miR-331-3p and PIK3R3 is undetermined, as well as their functions in OA progression.

In this study, we attempted to investigate the expression and role of circ_0045714, miR-331-3p and PIK3R3 in OA patients and IL-1 $\beta$-insulted human articular chondrocytes (HAC), and to further confirm the underlying relationship among them.

\section{Materials and methods}

\section{Cartilages and chondrocytes isolation}

OA knee articular cartilage samples and healthy cartilage samples were collected from 20 OA patients with total knee replacement and 20 trauma patients with amputation, respectively. Cartilage specimens were stored at $-80{ }^{\circ} \mathrm{C}$. OA patients were in accordance with clinical and radiological diagnostic criteria for OA, and experienced constant pain during the last three months. Trauma patients were without any arthritis. All patients were recruited from Wuhan General Hospital of People's Liberation Army, and each participator signed informed content. This study was ratified by the Ethics committee of this hospital.

Cartilages were minced into very small pieces and soaked into DMEM/F-12 medium (M23250; R\&D systems, Minneapolis, MN, USA) containing $0.1 \%$ trypsin (B81210; R\&D systems) and 0.2\% Collagenase II (MX1002-100MG; MKBio, Shanghai, China) at $37^{\circ} \mathrm{C}$ for $8 \mathrm{~h}$. Afterward, undigested tissues were removed using $40 \mathrm{~mm}$ filter, and cells were harvested by centrifugation. Isolated cells were cultured in DMEM/F-12 medium (R\&D systems) supplemented with $10 \%$ fetal bovine serum (FBS, S11150H; R\&D systems) and 1\% penicillinstreptomycin (B21210; R\&D systems). Complete medium was changed every three days and cells in 2 passage were harvested for use.

\section{Cell culture and transfection}

The immortalized HAC cell line (CHON-001; CRL-2846; ATCC, Manassas, VA, USA) was cultured in DMEM (M22650; R\&D systems) containing $0.1 \mathrm{mg} / \mathrm{ml} \mathrm{G418}$ disulfate salt (4131; R\&D systems) and 10\% FBS (R\&D systems). HAC cells were used to transiently transfect with exogenous nucleotides or vectors using Lipofectamine 3000 (Invitrogen, Carlsbad, CA, USA) for $36 \mathrm{~h}$. The oligonucleotides were circ_0045714-siRNA (si-circ_0045714), PIK3R3-siRNA (si-PIK3R3), negative control (NC)-siRNA (si-NC), miR-331-3p mimic, miRNC mimic, miR-331-3p inhibitor (anti-miR-331-3p), and miR-NC inhibitor (anti-miR-NC). The vectors were empty pCD5-ciR vector (GENESEED, Guangzhou, China), recombinant pCD5-ciR-circ_0045714 vector, pmiR-Reporter vector (Promega, Madison, WI, USA) expressing circ_0045714 containing the wild type (WT) or mutant type (MUT) of miR-331-3p response elements, and pmiR-Reporter vector (Promega, Madison, WI, USA) expressing PIK3R3 3'UTR containing WT or MUT of miR-331-3p response elements. Single and cotransfection models were performed per the instructions, and transfected cells at $36 \mathrm{~h}$ were harvested for further assays. 


\section{OA cell model induced by IL-1 $\beta$}

Transfected and un-transfected HAC cells in $80 \%$ confluence were starved in serum-free medium for $4 \mathrm{~h}$ and then replaced with complete medium added with $10 \mathrm{ng} / \mathrm{mL}$ IL-1 $\beta$ (Amyjet Scientific, Wuhan, China) for $24 \mathrm{~h}$. HAC cells without transfection or IL-1 $\beta$ treatment were served as control.

\section{Real-time quantitative polymerase chain reaction (RT-qPCR)}

RNA was isolated with TRIzol reagent (Life Technologies, Carlsbad, CA, USA) and reversely transcribed into cDNA with SuperScript II first-strand synthesis system (Invitrogen) for RT-qPCR. SYBR Green Mix (Qiagen, Germany) and StepOne ${ }^{\mathrm{TM}}$ Real-Time PCR System (Applied Biosystems, Carlsbad, CA, USA) were adopted for the PCR amplification and melting curve analysis. $2^{-\Delta \Delta C T}$ method was used to evaluate RNA expression level, and internal parameters glyceraldehyde-3-phosphate dehydrogenase (GAPDH) and U6 were used to correct RNA expression. Primers for circ_0045714, unk zinc finger (UNK), miR-331-3p and PIK3R3 are shown in Table 1.

\section{CircRNA characterization}

Actinomycin D and RNase R assays were implemented to measure the characterization of circ_0045714, comparing to its host gene UNK. Actinomycin D $(2 \mathrm{mg} / \mathrm{mL}$; Sigma-Aldrich, St. Louis, MO, USA) was added in HAC cells for $0,4,8,12$ and $24 \mathrm{~h}$, and RNA in each timing was isolated. RNA from $\mathrm{HAC}$ cells was treated with $3 \mathrm{U} / \mu \mathrm{g}$ RNase R (GENESEED) for $30 \mathrm{~min}$, and mock cells were without RNase R treatment. Expression of circ_0045714 and UNK was detected in Actinomycin D, RNase R and mock groups using RT-qPCR.

Table $1 \mathrm{qPCR}$ primers

\begin{tabular}{ll}
\hline & Primers $\left(\mathbf{5}^{\prime} \mathbf{-} \mathbf{3}^{\prime} \mathbf{)}\right.$ \\
\hline circ_0045714 (135nt) & Forward: CCATGTCCAAACGTCAAGCA \\
& Reverse: GTGGTTCTGTGCAGGAATGG \\
UNK (157nt) & Forward: AGCACTACACGTACCTGAAAGAT \\
& Reverse: TAATGAAGGTGCCGTCCCG \\
miR-331-3p (89nt) & Forward: ACACTCCAGCTGGGGCCCCTGGGCCTATC \\
& Reverse: CTCAACTGGTGTCGTGGAGTCGGCAAT \\
PIK3R3 (118nt) & Forward: GAGCTGACTGGACTTCTCCG \\
& Reverse: TGGTCTGCAGAGAGCGAATC \\
GAPDH (104nt) & Forward: GACAGTCAGCCGCATCTTCT \\
& Reverse: GCGCCCAATACGACCAAATC \\
U6 (89nt) & Forward: GCTTCGGCAGCACATATACTAAAAT \\
& Reverse: CGCTCACGAATTTGCTGTCAT \\
\hline
\end{tabular}

\section{Colony formation assay and cell viability assay}

A sum of 150 cells were seeded in 12-well plate and cultured for 15 days. Eventually, single cell was cloned into a colony, and cloned colonies were dyed with crystal violet method. Number of colonies ( $>30$ cells/colony) was manually determined under an inverted microscope. Cell Counting Kit-8 (CCK-8; Genomeditech, Shanghai, China) was employed to monitor cell viabilities of IL- $1 \beta$-treated HAC and control cells during 3 days by measuring the optical density (OD) values at $450 \mathrm{~nm}$.

\section{Apoptosis assay}

About $5 \times 10^{5}$ cells were co-incubated with Annexin V-fluorescein isothiocyanate (FITC) and propidium iodide (PI) according to the instructions of Annexin V-FITC/PI apoptosis detection kit (Yeasen, Guangzhou, China). Using flow cytometry (FCM), stained cells and unstained cells were analyzed: live cells were Annexin V-/ $\mathrm{PI}-$, early apoptotic cells were Annexin $\mathrm{V}+/ \mathrm{PI}-$, and late apoptotic cells and necrotic cells were Annexin $\mathrm{V}+/ \mathrm{PI}+$.

\section{Enzyme-linked immunosorbent assay (ELISA)}

The products of IL-6, IL- 8 and TNF- $\alpha$ in the cell culture supernatants were examined by the human IL-6 ELISA kit (EK0410; Boster, Pleasanton, CA, USA), IL-8 ELISA kit (EK0413; Boster) and TNF- $\alpha$ ELISA kit (EK0525; Boster), respectively. Four paralleled wells were for each group. Following the assay protocols, OD absorbance was read with a microplate reader at $450 \mathrm{~nm}$, and measured concentrations were determined using linear regression of OD value against the standard curve.

\section{Protein isolation}

Cellular protein was isolated from tissues and cells using RIPA lysis buffer (Beyotime, Shanghai, China), and culture medium protein was also isolated and concentrated via ultrafiltration membrane. Protein concentration was determined depending on BCA protein assay kit (Beyotime), and western blotting was performed in the following order: electrophoresis with polyacrylamide gel, membrane transferring via electrophoresis, antibody incubation using special primary antibodies (Table 2) and horseradish peroxidase (HRP)labeled secondary antibody. HRP signal was reacted with BeyoECL Plus kit (Beyotime), and then detected on chemiluminescence imaging system. Protein blots were analyzed on Image-pro-plus software (Media Cybernetics, Washington, USA).

\section{Dual-luciferase reporter assay}

HAC cells in exponential growth were co-transfected with the recombinant pmiR-Reporter vectors expressing 
Table 2 Primary antibodies in western blotting

\begin{tabular}{llll}
\hline Antibody & Cat. No & Dilution rate & Source \\
\hline BCl-2 & A00040-2 & $1: 500$ & Boster \\
Bax & M00183-1 & $1: 1000$ & Boster \\
Cleaved Casp3 & AC030 & $1: 1000$ & Beyotime \\
PIK3R3 & M06707 & $1: 1000$ & Boster \\
ADAMTS-5 & $\mathrm{A} 12210$ & $1: 100$ & Boster \\
Collagen II & PA2141 & $1: 500$ & Boster \\
GAPDH & M00227 & $1: 10000$ & Boster \\
\hline
\end{tabular}

WT/MUT-circ_0045714 or WT/MUT-PIK3R3 3'UTR and mimic of miR-NC or miR-331-3p for $36 \mathrm{~h}$. Cells were lysed and subjected to dual-luciferase reporter gene assay Kit (Beyotime). Luminescence was detected by luminometer. Relative light unit of Firefly luciferase was corrected by that of Renilla luciferase.

\section{Statistical analysis}

Data were presented in the form of mean \pm standard deviation. Each experiment was carried out in triplicate. One-way or two-way analysis of variance test followed by Tukey's post hoc test was used to determine the differences among groups on GraphPad Prism 7 (GraphPad,
La Jolla, CA, USA). $P$ value $<0.05$ was chosen to indicate a statistical significance.

\section{Results}

Down-regulation of circ_0045714 was one molecular event in OA patients and IL-1 $\beta$-insulted HAC cells

Circ_0045714 was derived from UNK via back-splicing event (Fig. 1A), and its expression was abundantly decreased in the knee articular cartilages and chondrocytes from OA patients than normal ones (Fig. 1B, C). Besides, comparing to the host gene UNK, circ_0045714 expression in HAC cells was little affected by actinomycin D treatment during $24 \mathrm{~h}$ (Fig. 1D) or RNase R treatment (Fig. 1E). These data showed that circ_0045714 was aberrantly down-regulated in OA patients and IL-1 $\beta$-insulted HAC.

\section{Hyper-expression of circ_0045714 relieved HAC} apoptosis, inflammatory response and matrix degradation under IL-1 $\beta$ stimulation with cell proliferation inhibition Genetic manipulation of circ_0045714 was carried out in OA cell model, and the expression of circ_0045714 in IL-1 $\beta$-insulted HAC cells was highly promoted via oe-circ_0045714 vector transfection (Fig. 2A). A series of functional experiments were further performed, and IL-1 $\beta$ led to a decrease in cell proliferation in HAC cells, as evidenced by the declined number of cloned

\section{A}
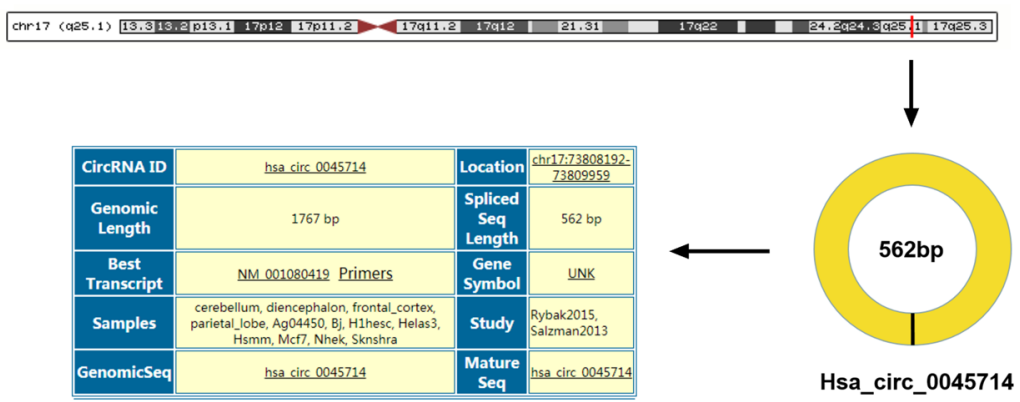

B

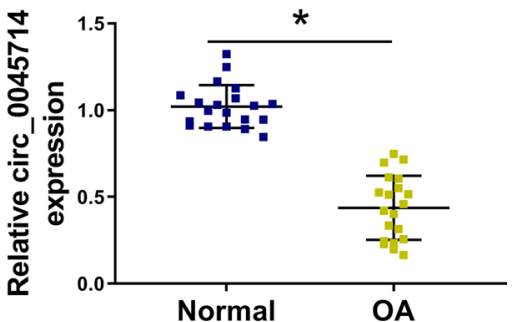

C

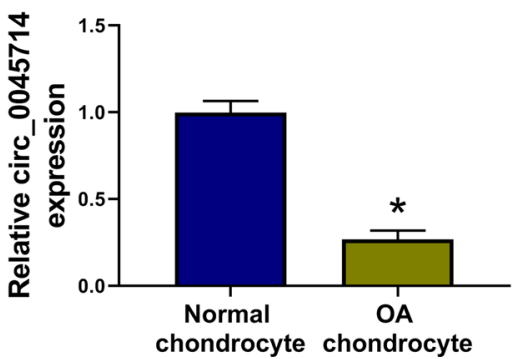

D

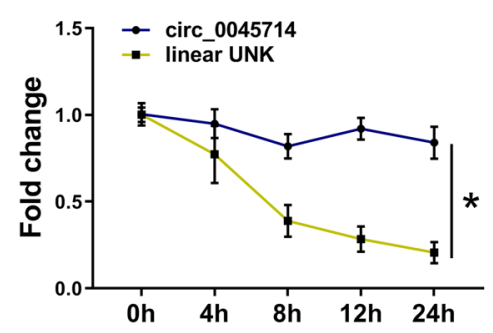

E

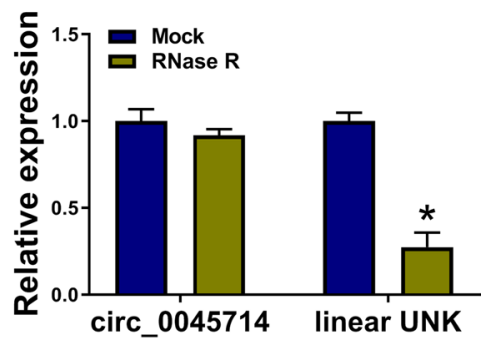

Fig. 1 Down-regulation of circ_0045714 was one molecular event in OA. A The information of circ_0045714 in genome mapping was shown. B, C RNA expression was analyzed by RT-qPCR, and relative circ_0045714 expression was determined in (B) OA cartilages ( $n=20)$ and normal cartilages $(n=20)$, and C OA chondrocytes and normal chondrocytes. D, E RNA expression was analyzed by RT-qPCR, and relative circ_0045714 and linear UNK expression was determined in HAC cells in actinomycin D group, RNase R group and mock group. ${ }^{*} P<0.05$ 


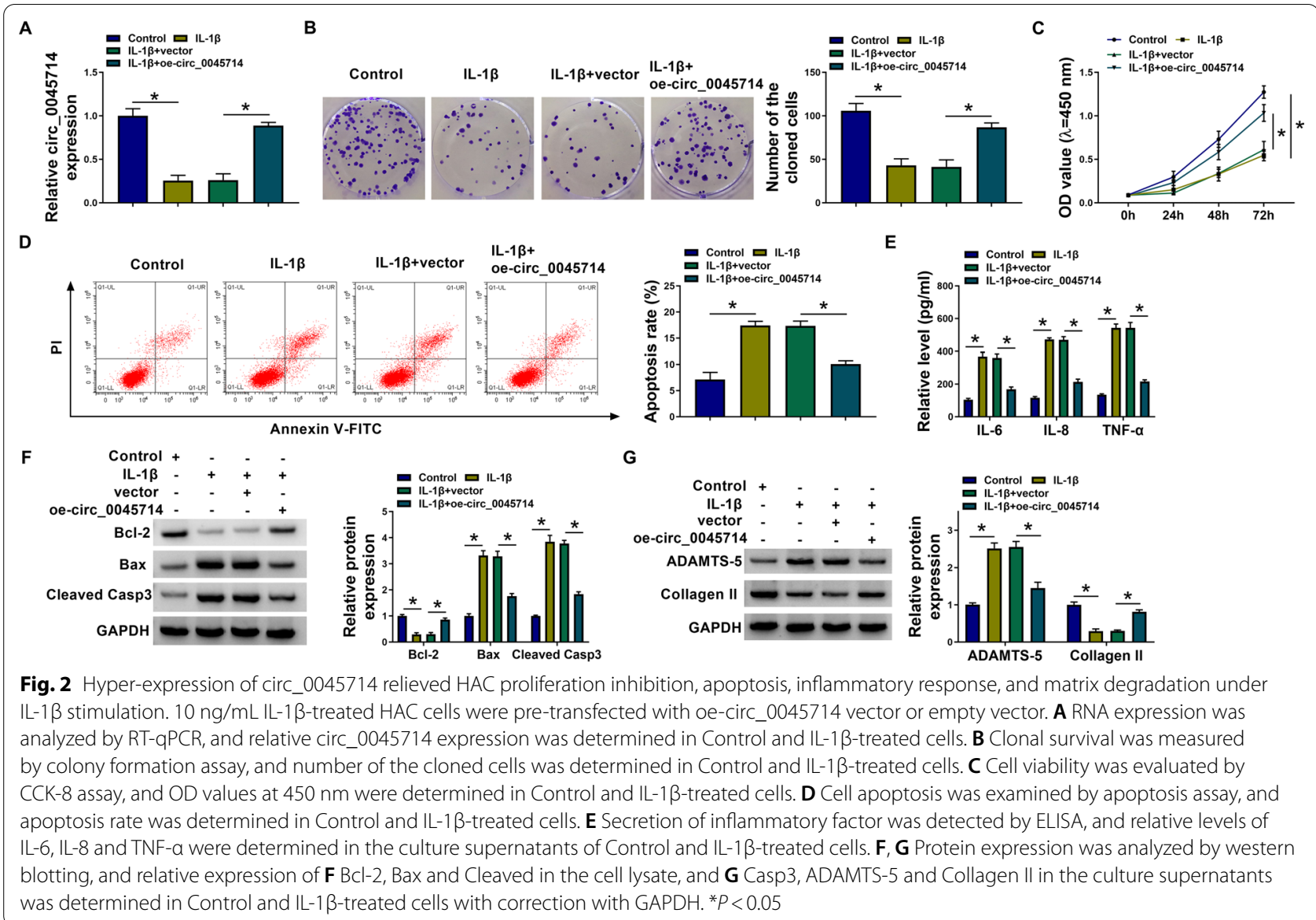

cells and cell viabilities (Fig. 2B, C). Notably, the presence of oe-circ_0045714 vector significantly improved the proliferation of IL-1 $\beta$-treated HAC cells (Fig. 2B, C). In contrast, apoptosis rate and expression of apoptosisrelated markers (Bax and cleaved Casp3) were sharply boosted under IL- $1 \beta$ stimulation, which were partially diminished due to oe-circ_0045714 vector-mediated circ_0045714 overexpression (Fig. 2D, F). IL-1 $\beta$ treatment evoked high production of pro-inflammatory factors (IL-6, IL- 8 and TNF- $\alpha$ ) and cartilage degradation marker (ADAMTS-5) in HAC cells, accompanying with low level of ECM component Collagen II (Fig. 2E, G), whereas IL-1 $\beta$-elicited inflammation and matrix degradation in HAC cells were mitigated by reinforcing circ_0045714 (Fig. 2E, G). These results demonstrated that overexpressing circ-0045714 promoted proliferation and matrix synthesis, but suppressed apoptosis and inflammatory response in HAC under IL- $1 \beta$ condition.

\section{MiR-331-3p was up-regulated in OA and functioned as a target for circ_0045714 in HAC cells}

According to the prediction result, miR-331-3p response elements in circ_0045714 were mutated for further confirmation (Fig. 3A). MiR-331-3p mimic caused overexpression of miR-331-3p in HAC cells (Fig. 3B), which then reduced the luciferase activity of WT-circ_0045714 reporter vector and left alone the mutant vector (Fig. 3C). Circ_0045714 expression was manipulated in HAC cells, and miR-331-3p level was gloomy or high when circ_0045714 was artificially overexpressed or silenced (Fig. 3D, E). In OA patients, miR-331-3p expression was elevated in the knee articular cartilages and chondrocytes (Fig. 3F, G). Moreover, Pearson correlation coefficient $(r)$ between circ_0045714 and miR-331-3p levels in these 20 OA cartilages was $-0.8030(P<0.0001$; Fig. $3 \mathrm{H})$. And, expression level of miR-331-3p in circ_0045714-overexpressed HAC cells under IL-1 $\beta$ stress was also promoted by its mimic (Fig. 4A). Functionally, circ_0045714 hyperexpression-mediated enhancement of colony formation and cell viability was abated by miR-331-3p mimic co-transfection (Fig. 4B, C); allied with this was the reverse of apoptosis rate and levels of IL-6, IL-8, TNF- $\alpha$, Bcl-2, Bax, Cleaved Casp3, ADAMTS-5, and Collagen II (Fig. 4D-G). These results indicated that miR-331-3p was up-regulated in human OA, and circ_0045714 functioned as a protective role in IL- $1 \beta$-evoked chondrocyte 

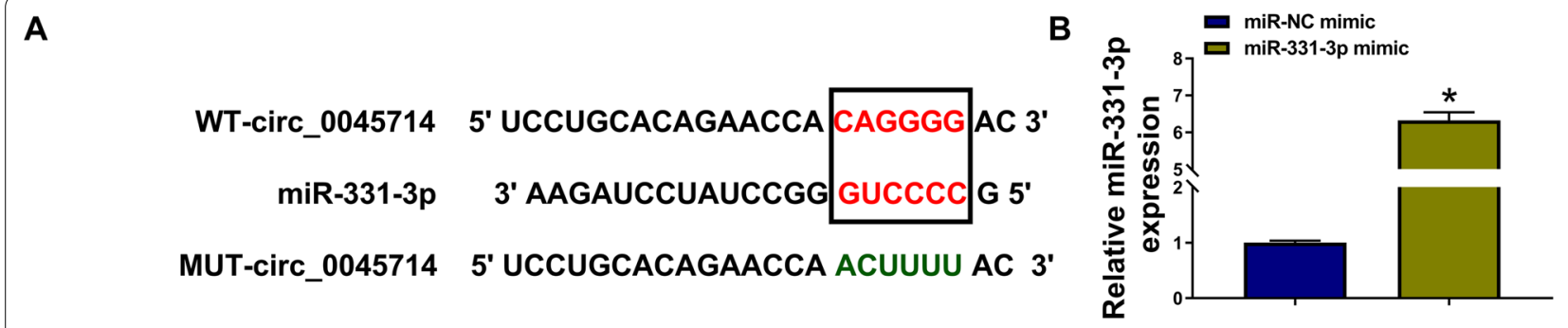

C

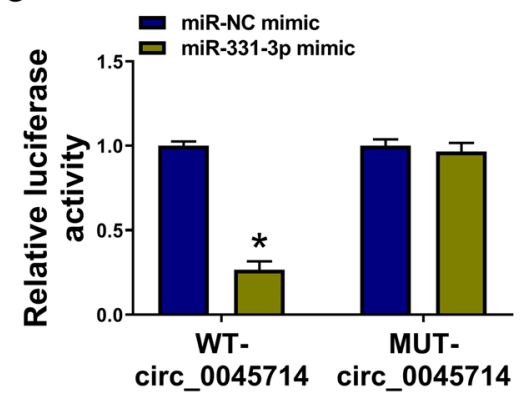

$\mathbf{F}$

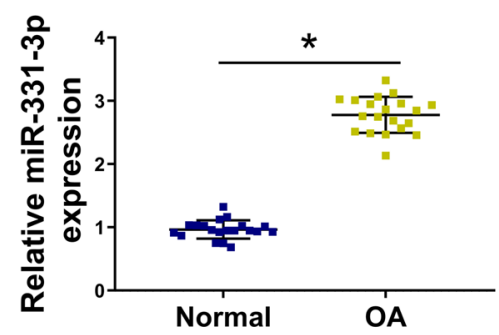

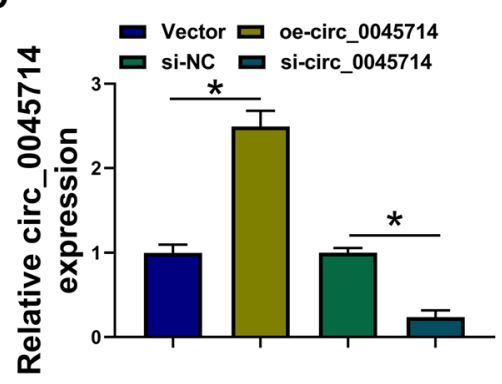

G

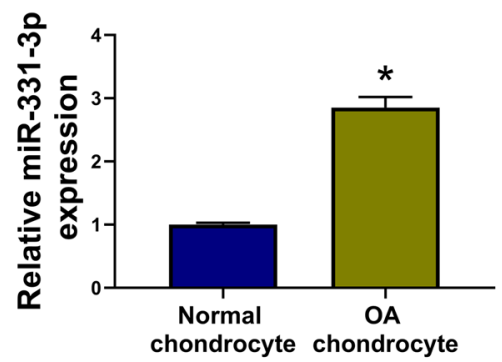

$\mathrm{E}$

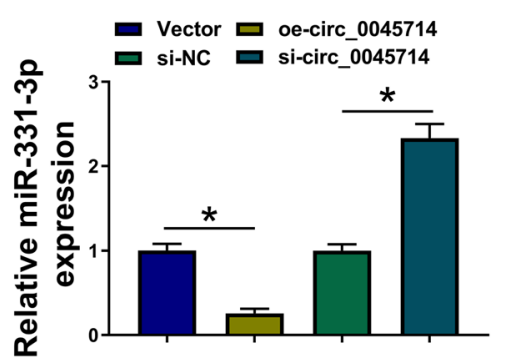

H

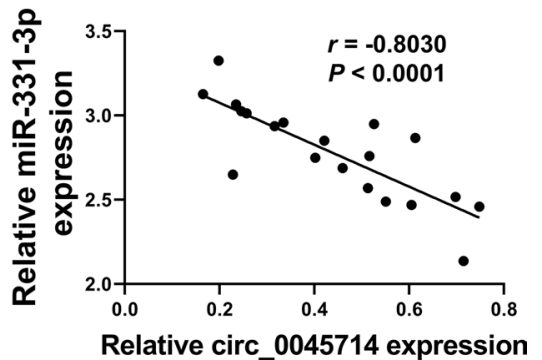

Fig. 3 Circ_0045714 sponged miR-331-3p expression via target binding. A The putative miR-331-3p-binding sites and their mutations were shown in circ_0045714. B RNA expression was analyzed by RT-qPCR, and relative miR-331-3p expression was determined in miR-NC or miR-331-3p mimic-transfected HAC cells. C Luciferase activity of reporter vector was measured by dual-luciferase reporter assay, and relative luciferase activity of WT-circ_0045714 and MUT-circ_0045714 was determined in HAC cells co-transfected with miR-NC or miR-331-3p mimic. D, E RNA expression was analyzed by RT-qPCR, and relative circ_0045714 and miR-331-3p expression was determined in HAC cells transfected with oe-circ_0045714 vector, empty vector, si-circ_0045714, or si-NC. F, G RNA expression was analyzed by RT-qPCR, and relative miR-331-3p expression was determined in OA cartilages $(n=20)$ and chondrocytes and normal cartilages $(n=20)$ and chondrocytes from patients. $\mathbf{H}$ Linear correlation between two variables was analyzed using Pearson correlation test, and the correlation coefficient ( $r$ ) between circ_0045714 and miR-331-3p in OA cartilages $(n=20)$ was determined. ${ }^{*} P<0.05$

injury through negatively regulating miR-331-3p via target binding.

\section{Exhausting miR-331-3p suppressed IL-1 $\beta$-evoked HAC} injury through up-regulating PIK3R3 via target binding PIK3R3 was predicted to show miR-331-3p response elements at 3'UTR (Fig. 5A). Moreover, dual-luciferase reporter assay identified the responsiveness of WTPIK3R3 3'UTR vector to miR-331-3p overexpression mediated by the mimic (Fig. 5B). In HAC cells, protein level of PIK3R3 was facilitated or depressed in condition of miR-331-3p inhibition via inhibitor transfection or overexpression via mimic transfection (Fig. 5C,
D). PIK3R3 expression at both mRNA and protein levels was lower in knee cartilages and chondrocytes isolated from OA patients than normal ones from normal patients (Fig. 5E-G). As determined by Pearson correlation test, the coefficient $(r)$ between miR-331-3p and PIK3R3 mRNA levels in 20 OA cartilages was -0.8077 $(P<0.0001$; Fig. $5 \mathrm{H})$. These results showed that PIK3R3 was abnormally down-regulated in OA through serving as downstream target gene for miR-331-3p, suggesting an interactive effect between miR-331-3p and PIK3R3 in OA progression.

Functional experiments were performed in IL-1 $\beta$ induced OA model by introducing anti-miR-331-3p 

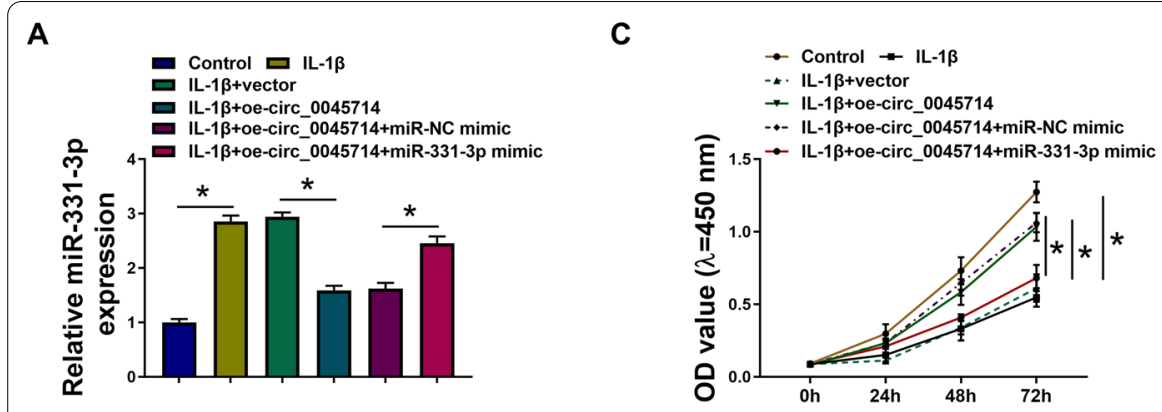

B

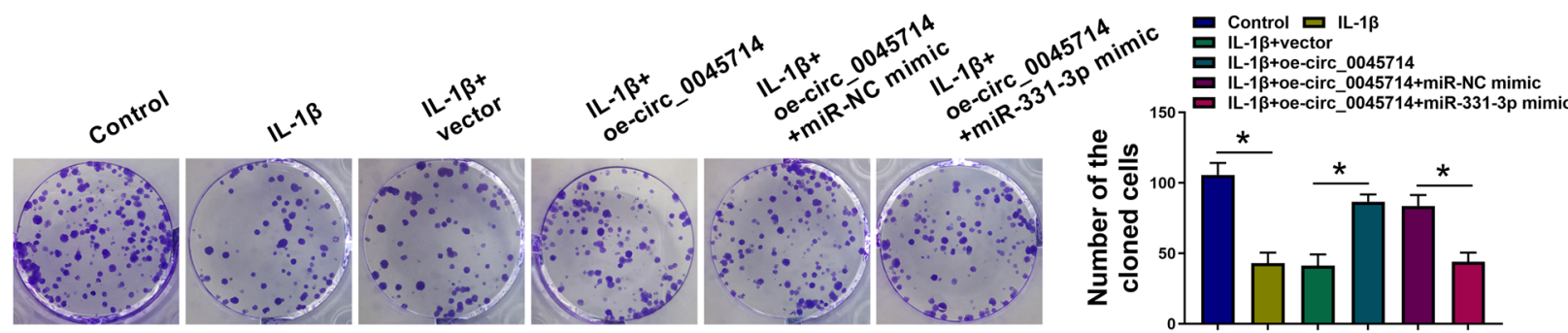

D
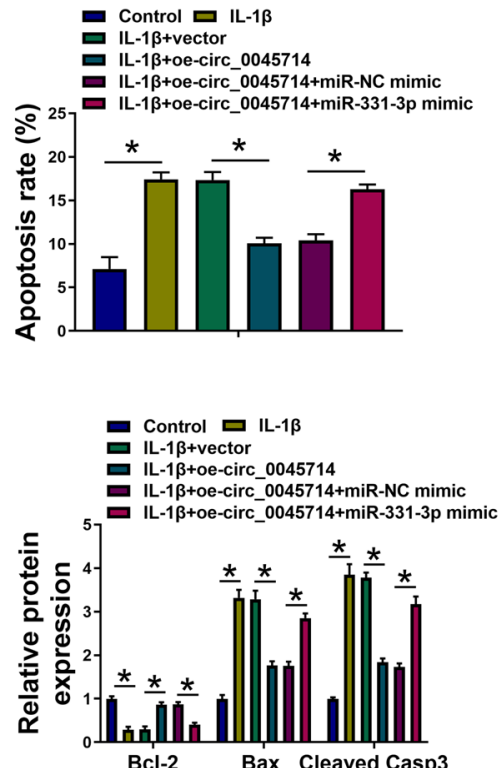

E

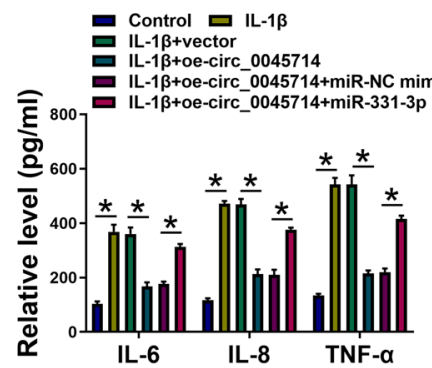

G

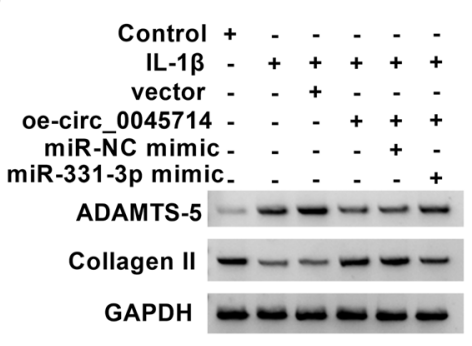

$\mathbf{F}$

Control + - - - - -

$\mathrm{IL}-1 \beta-++++$

vector - - + - - -

oe-circ_0045714 - - - + + +

miR-NC mimic- - - - + -

miR-331-3p mimic - - - +

$\mathrm{Bcl}-2=---1$

Bax - - - - -

Cleaved Casp3 $--\square--$

GAPDH $=-\infty-\infty$

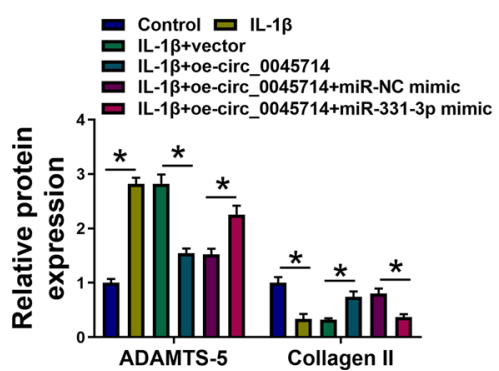

Fig. 4 Restoring miR-331-3p counteracted the roles of circ_0045714 in IL-1 $\beta$-insulted HAC. 10 ng/mL IL-1 $\beta$-treated HAC cells were pre-transfected with empty vector, oe-circ_0045714 vector, or oe-circ_0045714 vector coupled with miR-NC or miR-331-3p mimic. In Control and IL-1 $\beta$-treated cells, $\mathbf{A}$ relative miR-331-3p expression was detected by RT-qPCR, $\mathbf{B}$ number of the cloned cells was determined by colony formation assay, $\mathbf{C}$ OD values at $450 \mathrm{~nm}$ were measured by CCK-8 assay, D apoptosis rate was examined by apoptosis assay, E relative levels of IL-6, IL-8 and TNF-a in the culture supernatant were detected by ELISA, F, G relative expression of BCl-2, Bax, Cleaved Casp3, ADAMTS-5, and Collagen II in cell lysate or cell culture supernatant was determined by western blotting with correction with GAPDH. ${ }^{*} P<0.05$

alone or together with si-PIK3R3. As a result, PIK3R3 protein expression was inhibited in IL-1 $\beta$-treated HAC cells, and endogenously silencing miR-331-3p rescued PIK3R3 level (Fig. 6A); meanwhile, PIK3R3 expression in miR-331-3p-silenced cells under IL-1 $\beta$ stimulation could be blocked by its siRNA (Fig. 6A). Colony number and cell viability of IL-1 $\beta$-insulted HAC cells were accelerated with inhibiting miR-331-3p (Fig. 6B, C), and this acceleration was significantly weakened by inhibiting PIK3R3 (Fig. 6B, C). Contrarily, IL-1 $\beta$-elicited apoptosis rate promotion and high level of IL-6, IL-8, TNF- $\alpha$, Bax, and Cleaved Casp3 in HAC cells were restrained by 


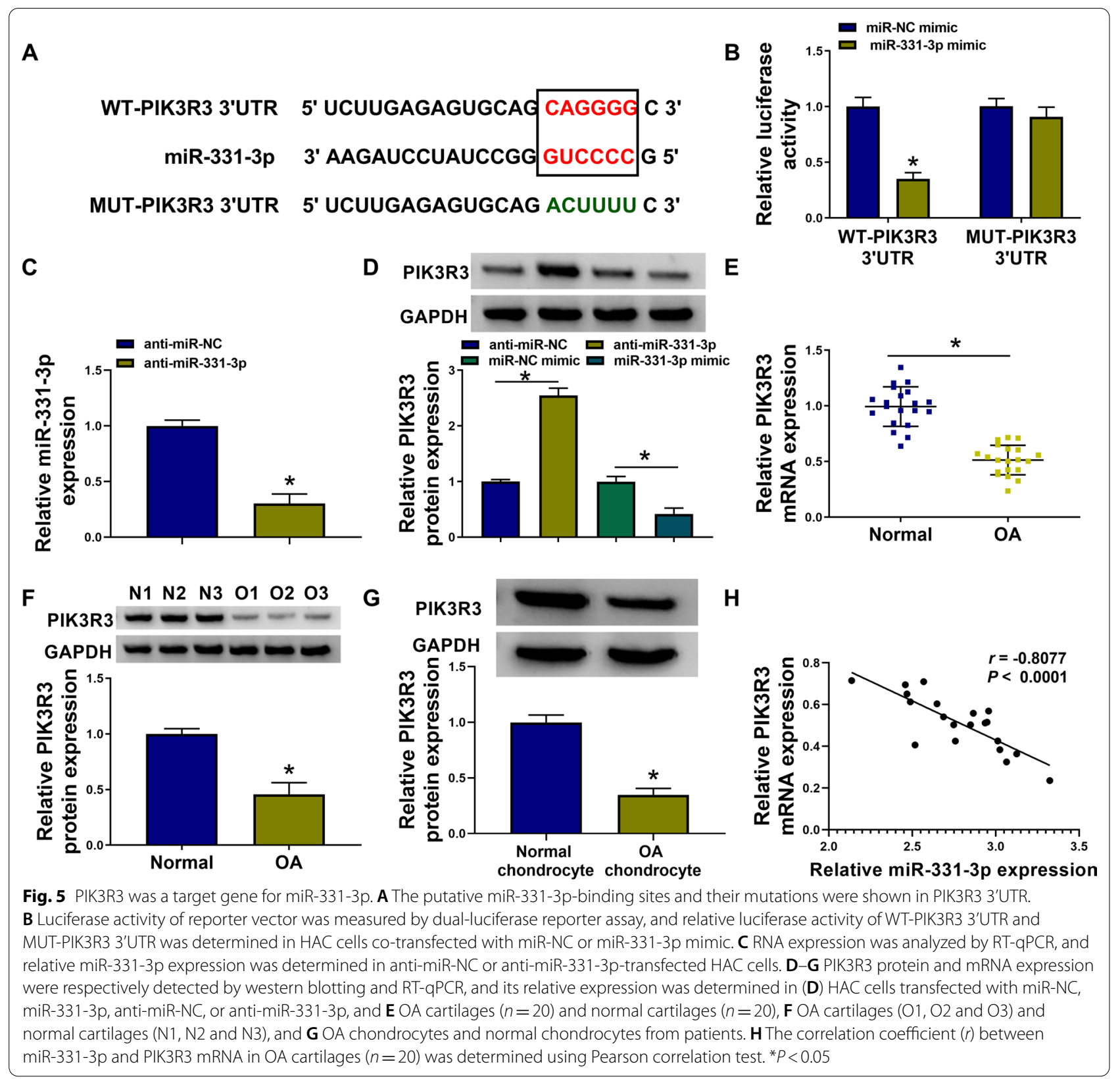

transfecting anti-miR-331-3p, and si-PIK3R3 could cause a disinhibition for that (Fig. 6D-F). Additionally, matrix degradation (ADAMTS-5 up-regulation and Collagen II down-regulation) was induced by IL-1 $\beta$ in HAC cells, which was suppressed by silencing miR-331-3p (Fig. 6G); interfering PIK3R3 almost neutralized the effect of miR331-3p inhibition on cartilage degradation under IL-1 $\beta$ stress (Fig. 6G). These outcomes demonstrated a counteractive role between miR-331-3p and PIK3R3 in regulating IL-1 $\beta$-elicited HAC cell proliferation inhibition, apoptosis, inflammation, and ECM degradation.
There was an interaction between circ_0045714 and PIK3R3 via miR-331-3p

Intriguingly, there was a linear correlation between circ_0045714 and PIK3R3 mRNA levels in these 20 OA cartilages $(r=0.7857, P<0.0001$; Fig. 7A). In HAC cells, circ_0045714 mediated a positive regulatory effect on PIK3R3 protein expression, and this regulation was affected by the alteration of miR-331-3p level (Fig. 7B, C). This data showed an interaction among circ_0045714, miR-331-3p and PIK3R3 in HAC in OA. 

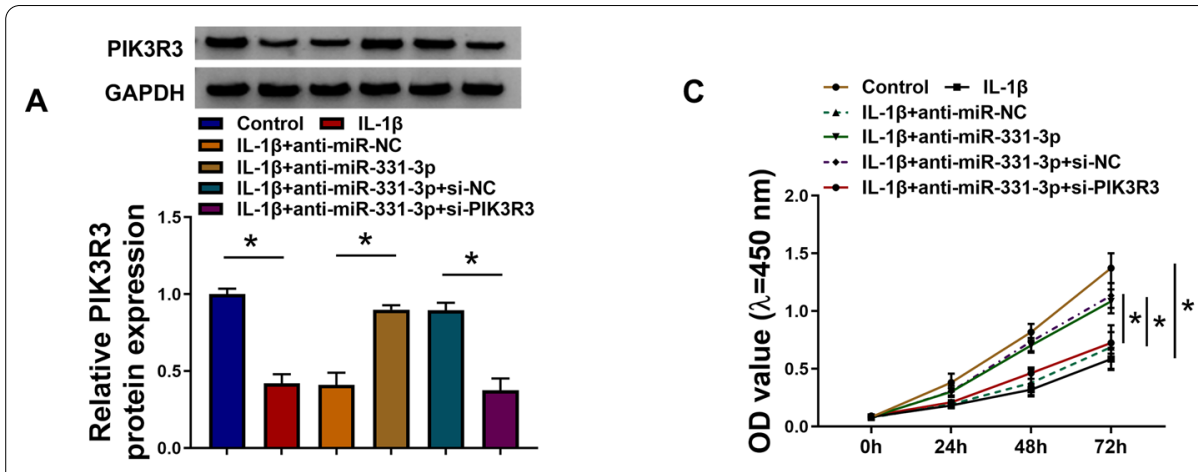

B

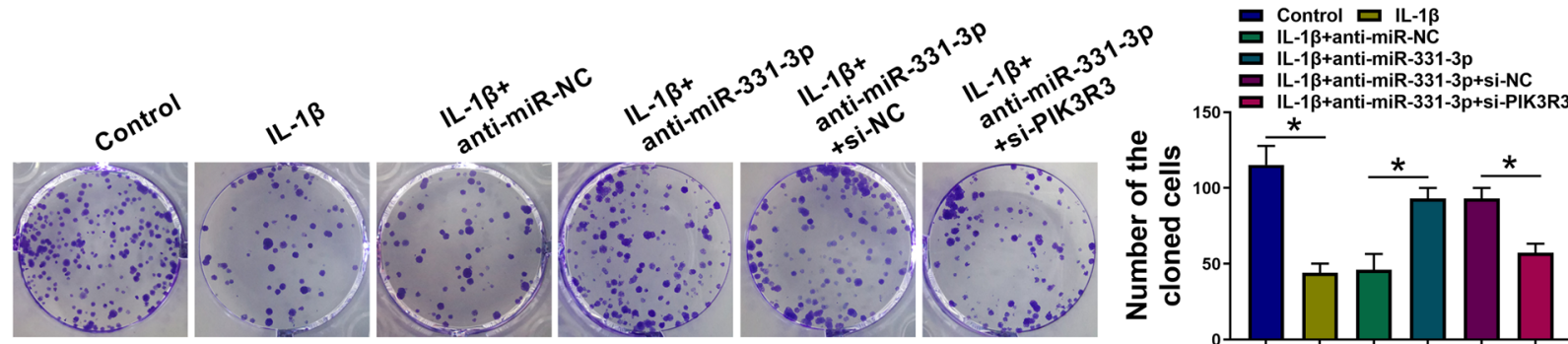

D
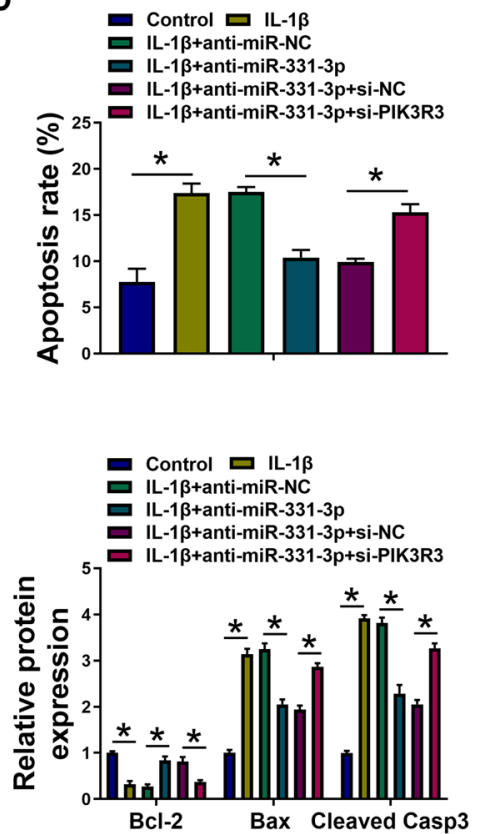

E

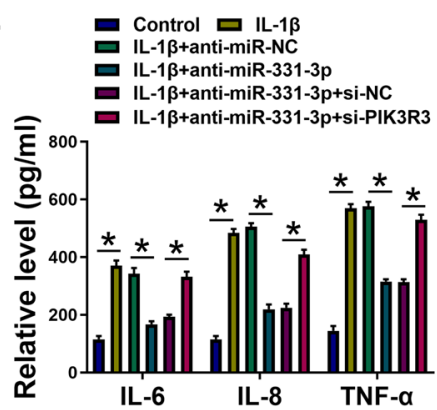

F
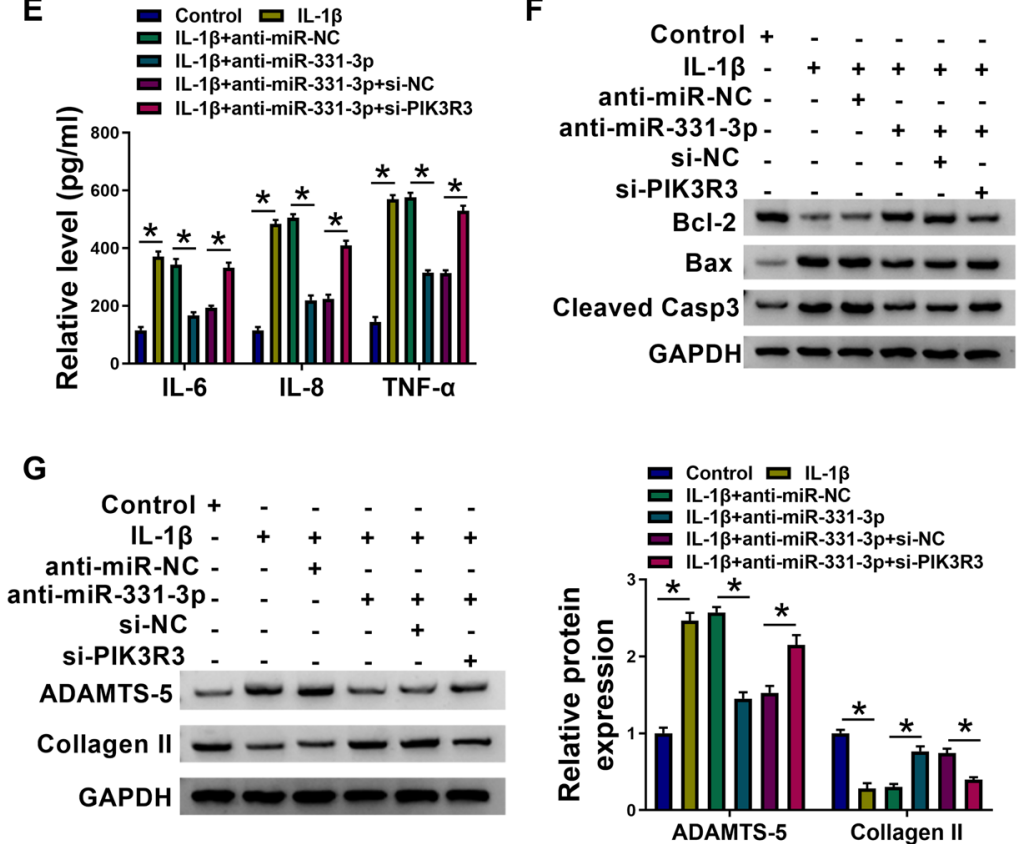

Fig. 6 Exhausting PIK3R3 neutralized the functions of miR-331-3p inhibition in IL-1 $\beta$-insulted HAC. $10 \mathrm{ng} / \mathrm{mL}$ IL-1 $\beta$-treated HAC cells were pre-transfected with anti-miR-NC, anti-miR-331-3p, or anti-miR-331-3p coupled with si-NC or si-PIK3R3. In Control and IL-1 $\beta$-treated cells, $\mathbf{A}$ relative PIK3R3 protein expression was detected by western blotting, $\mathbf{B}$ number of the cloned cells was determined by colony formation assay, $\mathbf{C}$ OD values at $450 \mathrm{~nm}$ were measured by CCK-8 assay, D apoptosis rate was examined by apoptosis assay, E relative levels of IL-6, IL-8 and TNF-a in the culture supernatant were detected by ELISA, F, G relative expression of BCl-2, Bax, Cleaved Casp3, ADAMTS-5, and Collagen II in cell lysate or cell culture supernatant was determined by western blotting with correction with GAPDH. ${ }^{*} P<0.05$

\section{Discussion}

CircRNAs have been proved to serve as contributors or interrupters in the pathogenesis of OA via ceRNA pathway in HAC, such as ciRS-7 [27]. In this study, we found that circ_0045714 was an interrupter in IL-1 $\beta$-induced OA development, and its hyper-expression suppressed $\mathrm{HAC}$ apoptosis and inflammation, and improved the proliferation and ECM synthesis under IL-1 $\beta$ stimulation 

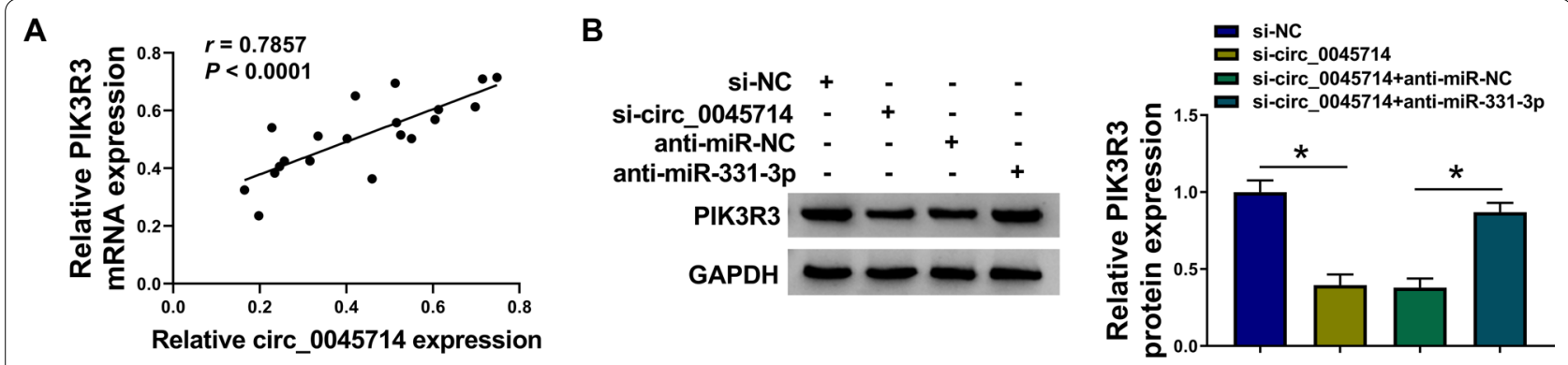

C

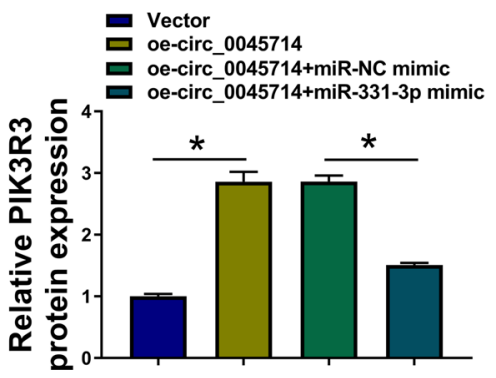

Fig. 7 There was an interaction between circ_0045714 and PIK3R3 via miR-331-3p. A The correlation coefficient ( $r$ ) between circ_0045714 and PIK3R3 mRNA in OA cartilages $(n=20)$ was determined by Pearson correlation test. B, C Western blotting detected relative PIK3R3 protein expression in HAC cells transfected with si-NC, si-circ_0045714, or si-circ_0045714 coupled with anti-miR-NC or anti-miR-331-3p, empty vector, oe-circ_0045714 vector, or oe-circ_0045714 vector coupled with miR-NC or miR-331-3p mimic. ${ }^{*} P<0.05$

through targeting miR-331-3p and regulating PIK3R3, the inhibitor of PI3K signal.

Here, circ_0045714 expression was found to be lower in both knee cartilage tissues and cells from OA patients than normal controls, which was in favor with previous data $[14,28]$. Besides, we discovered a down-regulation of circ_0045714 in IL-1 $\beta$-insulted HAC, and this finding was consistent with the finding in TNF- $\alpha$-induced OA model in HAC $[15,28]$. Moreover, we noticed that circ_0045714 was resistant to RNase R digestion and actinomycin D (a transcriptional inhibitor) treatment. Gain-of-functional experiments revealed that circ_0045714 re-expression elevated colony formation and cell viability of IL-1 $\beta$-treated HAC, and suppressed apoptosis (lower apoptosis rate and expression of Bax and cleaved Casp3), inflammatory response (higher IL-6, IL-8 and TNF- $\alpha$ contents), and matrix degradation (lower ADAMTS-5 and higher Collagen II), suggesting a protective role of circ_0045714 in IL-1 $\beta$-induced OA in HAC. Accidently, Fang et al. [16] also proposed that circ_0045714 up-regulation could be the molecular mechanism of the relief of OA both in vitro and in vivo. Moreover, reinforcing circ_0045714 had been previously demonstrated to alleviate HAC under TNF- $\alpha$ stress via regulating the proliferation, apoptosis, ECM synthesis, and inflammation $[15,28]$. Classically, we identified a novel ceRNA axis of circ_0045714 via targeting miR331-3p and modulating PIK3R3.
MiR-331-3p was an inhibitor of inflammatory response in different inflammation-related diseases [29-31], including rheumatoid arthritis [32]. Here, the expression of miR-331-3p was highly induced in OA samples, including knee cartilages, ex vivo chondrocytes, and IL- $1 \beta$-insulted HAC cells. This might be a pioneer study of miR-331-3p in human OA, and we found that inhibiting miR-331-3p might prevent HAC cells from IL-1 $\beta$-evoked damages via circ_0045714/miR-331-3p/ PIK3R3 axis. PIK3R3 was previously documented to be down-regulated in OA tissues and functioned as a target of miR-1236 [33]. Similarly, our data described a lower level of PIK3R3 in OA knee cartilages and ex vivo cartilage cells, as well as in vitro HAC under IL- $1 \beta$ stress. In addition, PIK3R3 was directly targeted by miR-331-3p in regulation of not only HAC cell apoptosis, but also the proliferation, inflammatory response and matrix degradation. Even though PIK3R3 was a key gene in PI3K/ AKT signaling pathway [34,35] and this way had been well-known in OA chondrocytes [36, 37], the association between PIK3R3 and chondrocyte injury was still not been elaborated yet. However, we enhanced the knowledge of RNA interference of PIK3R3 via miR-331-3p and its siRNA.

Except for PIK3R3, we also noticed that PIK3R2 and PIK3R5 were predicted to have miR-331-3p response elements according to starBase software (http://starb ase.sysu.edu.cn/hsa-miR-331-3p\&PIK3Rs). However, 
the potential target relationship between miR-331-3p and PIK3R2 or PIK3R5 was not further confirmed. By the way, PI3K/AKT pathway was the downstream of miR-155/PIK3R1 axis in dictating the cell fate of chondrocytes under IL-1 $\beta$ condition [38], and autophagy was affected by PI3K/AKT pathway in OA chondrocytes [27, 36]; however, PI3K/AKT pathway and autophagy were left to be further studied underlying circ_0045714/miR-331-3p/PIK3R3 axis.

\section{Conclusion}

In conclusion, circ_0045714 and PIK3R3 were down-regulated and miR-311-3p was up-regulated in OA patients, and restoring circ_0045714 could prevent HAC against IL-1 $\beta$-elicited cell apoptosis, inflammatory response and ECM degradation by regulating miR-331-3p and PIK3R3 via a ceRNA axis. Moreover, circ_0045714/miR-331-3p axis might mediate inhibition of PI3K/AKT signaling pathway via targeting PIK3R3.

\section{Abbreviations}

OA: Osteoarthritis; circ 0045714: Hsa circ 0045714; HAC: Human articular chondrocytes; PIK3R3: Phosphoinositide-3-kinase regulatory subunit 3; IL: Interleukin; TNF: Tumor necrosis factor; Bax: Bcl-2-associated X protein; ADAMTS: A disintegrin-like and metallopeptidase with thrombospondin motif; ceRNA: Competing endogenous RNA.
\end{abstract}

\section{Acknowledgements}

None.

\section{Authors' contributions}

$J X$ and $H L$ designed and performed the research; TZ, XX and ZS analyzed the data; RD and JZ wrote the manuscript. All authors read and approved the final manuscript.

\section{Funding}

None.

\section{Availability of data and materials}

Not applicable.

\section{Declarations}

\section{Ethics approval and consent to participate}

Written informed consents were obtained from all participants, and this study was permitted by the Ethics Committee of Wuhan General Hospital of People's Liberation Army.

\section{Consent for publication}

Not applicable.

\section{Competing interests}

The authors declare that they have no competing interests.

\section{Author details}

${ }^{1}$ Department of Orthopedic Surgery, Wuhan General Hospital of People's Liberation Army, Wuhan, China. ${ }^{2}$ Department of Pain1, Hubei Provincial Hospital of Traditional Chinese Medicine, 856 Luoyu Road, Hongshan District, Wuhan City 430070, Hubei Province, China.
Received: 12 July 2021 Accepted: 21 September 2021

Published online: 14 October 2021

\section{References}

1. Ratneswaran A, Kapoor M. Osteoarthritis year in review: genetics, genomics, epigenetics. Osteoarthr Cartil. 2021;29(2):151-60.

2. Neogi T. The epidemiology and impact of pain in osteoarthritis. Osteoarthr Cartil. 2013;21(9):1145-53.

3. Hawker GA, Stewart L, French MR, Cibere J, Jordan JM, March L, SuarezAlmazor M, Gooberman-Hill R. Understanding the pain experience in hip and knee osteoarthritis - an OARSI/OMERACT initiative. Osteoarthr Cartil. 2008;16(4):415-22.

4. Moilanen $\sqcup$, Hamalainen M, Nummenmaa E, Ilmarinen P, Vuolteenaho K, Nieminen RM, Lehtimaki L, Moilanen E. Monosodium iodoacetateinduced inflammation and joint pain are reduced in TRPA1 deficient mice-potential role of TRPA1 in osteoarthritis. Osteoarthr Cartil. 2015;23(11):2017-26.

5. Tchetina EV, Glemba KE, Markova GA, Naryshkin EA, Taskina EA, Makarov MA, Lila AM. Development of postoperative pain in patients with endstage knee osteoarthritis is associated with upregulation of genes related to extracellular matrix degradation, inflammation, and apoptosis measured in the peripheral blood before knee surgery. Life. 2020;10(10):224.

6. Aigner T, Kurz B, Fukui N, Sandell L. Roles of chondrocytes in the pathogenesis of osteoarthritis. Curr Opin Rheumatol. 2002;14(5):578-84.

7. Glyn-Jones S, Palmer AJ, Agricola R, Price AJ, Vincent TL, Weinans H, Carr AJ. Osteoarthritis. Lancet. 2015;386(9991):376-87.

8. Robinson WH, Lepus CM, Wang Q, Raghu H, Mao R, Lindstrom TM, Sokolove J. Low-grade inflammation as a key mediator of the pathogenesis of osteoarthritis. Nat Rev Rheumatol. 2016;12(10):580-92.

9. Jenei-Lanzl Z, Meurer A, Zaucke F. Interleukin-1 beta signaling in osteoarthritis - chondrocytes in focus. Cell Signal. 2019;53:212-23.

10. Razmara E, Bitaraf A, Yousefi H, Nguyen TH, Garshasbi M, Cho WC, Babashah S. Non-coding RNAs in cartilage development: an updated review. Int J Mol Sci. 2019;20(18):4475.

11. Jiang $S$, Liu Y, Xu B, Zhang Y, Yang M. Noncoding RNAs: new regulatory code in chondrocyte apoptosis and autophagy. Wiley Interdiscip Rev RNA. 2020;11(4):e1584.

12. Liu D, Liang YH, Yang YT, He M, Cai ZJ, Xiao WF, Li YS. Circular RNA in osteoarthritis: an updated insight into the pathophysiology and therapeutics. Am J Transl Res. 2021;13(1):11-23.

13. Sun Y, Ge J, Tang W, Hong H, Liu D, Lin J. Hsa_circ_0045714 induced by eupatilin has a potential to promote fracture healing. BioFactors. 2021:47:376-85.

14. Xiao K, Xia Z, Feng B, Bian Y, Fan Y, Li Z, Wu Z, Qiu G, Weng X. Circular RNA expression profile of knee condyle in osteoarthritis by illumina HiSeq platform. J Cell Biochem. 2019;120(10):17500-11.

15. Li BF, Zhang Y, Xiao J, Wang F, Li M, Guo XZ, Xie HB, Xia H, Chen B. Hsa_circ_0045714 regulates chondrocyte proliferation, apoptosis and extracellular matrix synthesis by promoting the expression of miR-193b target gene IGF1R. Hum Cell. 2017;30(4):311-8.

16. Fang $L$, Lin $L, L v Y$, Huang $Z$, Lin $X$, Wang $X$, Chen B. The mechanism of aerobic exercise combined with glucosamine therapy and circUNK in improving knee osteoarthritis in rabbits. Life Sci. 2021;275:119375.

17. Sun K, Luo J, Guo J, Yao X, Jing X, Guo F. The PI3K/AKT/mTOR signaling pathway in osteoarthritis: a narrative review. Osteoarthr Cartil. 2020;28(4):400-9.

18. Liu Z, Hong Z, Ma H, Yu D, Qu P. Key factors mediated by PI3K signaling pathway and related genes in endometrial carcinoma. J Bioenerg Biomembr. 2020;52(6):465-73.

19. Ge Y, Liu H, Qiu X, Ma G, Wang H, Du M, Wang M, Zhao Q, Tao G, Chu H, et al. Genetic variants in PI3K/Akt/mTOR pathway genes contribute to gastric cancer risk. Gene. 2018;670:130-5.

20. Rai MF, Pan H, Yan H, Sandell LJ, Pham CTN, Wickline SA. Applications of RNA interference in the treatment of arthritis. Transl Res. 2019;214:1-16.

21. Shi Q, Zhang XL, Dai KR, Benderdour M, Fernandes JC. siRNA therapy for cancer and non-lethal diseases such as arthritis and osteoporosis. Expert Opin Biol Ther. 2011;11(1):5-16.

22. Gargano G, Oliviero A, Oliva F, Maffulli N. Small interfering RNAs in tendon homeostasis. Br Med Bull. 2021;138(1):58-67. 
23. Giordano L, Porta GD, Peretti GM, Maffulli N. Therapeutic potential of microRNA in tendon injuries. Br Med Bull. 2020;133(1):79-94.

24. Oliviero A, Della Porta G, Peretti GM, Maffulli N. MicroRNA in osteoarthritis: physiopathology, diagnosis and therapeutic challenge. Br Med Bull. 2019;130(1):137-47.

25. Ghafouri-Fard S, Abak A, Tondro Anamag F, Shoorei H, Majidpoor J, Taheri $M$. The emerging role of non-coding RNAs in the regulation of PI3K AKT pathway in the carcinogenesis process. Biomed Pharmacother Biomed Pharmacother. 2021;137:111279.

26. Zhao D, Sui Y, Zheng X. MiR-331-3p inhibits proliferation and promotes apoptosis by targeting HER2 through the PI3K/Akt and ERK1/2 pathways in colorectal cancer. Oncol Rep. 2016;35(2):1075-82.

27. Zhou X, Li J, Zhou Y, Yang Z, Yang H, Li D, Zhang J, Zhang Y, Xu N, Huang $Y$, et al. Down-regulated ciRS-7/up-regulated miR-7 axis aggravated cartilage degradation and autophagy defection by PI3K/AKT/mTOR activation mediated by IL-17A in osteoarthritis. Aging. 2020;12(20):20163-83.

28. Jiang H, Dai J, Zhang C, Sun H, Tang X. Circ_0045714 alleviates TNFalpha-induced chondrocyte injury and extracellular matrix degradation through miR-218-5p/HRAS axis. J Bioenerg Biomembr. 2021;53(1):97-107.

29. Zhang X, Guo H, Xie A, Liao O, Ju F. microRNA-331-3p attenuates neuropathic pain following spinal cord injury via targeting RAP1A. J Biol Regul Homeost Agents. 2020;34(1):25-37.

30. Nie H, Hu Y, Guo W, Wang W, Yang Q, Dong Q, Tang Y, Li Q, Tang Z. miR331-3p Inhibits inflammatory response after intracerebral hemorrhage by directly targeting NLRP6. Biomed Res Int. 2020;2020:6182464.

31. Liu Q, Lei C. Neuroprotective effects of miR-331-3p through improved cell viability and inflammatory marker expression: correlation of serum miR-331-3p levels with diagnosis and severity of Alzheimer's disease. Exp Gerontol. 2021;144:111187.
32. Zhao Q, Zhao F, Liu C, Xu T, Song K. LncRNA FOXD2-AS1 promotes cell proliferation and invasion of fibroblast-like synoviocytes by regulation of miR-331-3p/PIAS3 pathway in rheumatoid arthritis. Autoimmunity. 2021;54:254-63.

33. Wang WT, Huang ZP, Sui S, Liu JH, Yu DM, Wang WB. microRNA-1236 promotes chondrocyte apoptosis in osteoarthritis via direct suppression of PIK3R3. Life Sci. 2020;253:117694.

34. Xu W, Yu M, Qin J, Luo Y, Zhong M. LACTB regulates PIK3R3 to promote autophagy and inhibit EMT and proliferation through the PI3K/AKT/ mTOR signaling pathway in colorectal cancer. Cancer Manag Res. 2020;12:5181-200.

35. Yuan L, Fan L, Li Q, Cui W, Wang X, Zhang Z. Inhibition of miR-181 b-5p protects cardiomyocytes against ischemia/reperfusion injury by targeting AKT3 and PI3KR3. J Cell Biochem. 2019;120(12):19647-59.

36. He W, Cheng Y. Inhibition of miR-20 promotes proliferation and autophagy in articular chondrocytes by PI3K/AKT/mTOR signaling pathway. Biomed Pharmacother Biomed Pharmacother. 2018;97:607-15.

37. Wen X, Li H, Sun H, Zeng A, Lin R, Zhao J, Zhang Z. MiR-455-3p reduces apoptosis and alleviates degeneration of chondrocyte through regulating PI3K/AKT pathway. Life Sci. 2020;253:117718.

38. Fan Z, Liu Y, Shi Z, Deng K, Zhang H, Li Q, Cao S, Li S, Zhang H. MiR-155 promotes interleukin-1 beta-induced chondrocyte apoptosis and catabolic activity by targeting PIK3R1-mediated PI3K/Akt pathway. J Cell Mol Med. 2020;24(15):8441-51.

\section{Publisher's Note}

Springer Nature remains neutral with regard to jurisdictional claims in published maps and institutional affiliations.
Ready to submit your research? Choose BMC and benefit from:

- fast, convenient online submission

- thorough peer review by experienced researchers in your field

- rapid publication on acceptance

- support for research data, including large and complex data types

- gold Open Access which fosters wider collaboration and increased citations

- maximum visibility for your research: over $100 \mathrm{M}$ website views per year

At BMC, research is always in progress.

Learn more biomedcentral.com/submissions 